\title{
Vortex field simulation and analysis of a solar updraft power engine
}

\author{
H. H. Al-Kayiem ${ }^{1}$, A. T. Mustafa ${ }^{2}$ \& S. I. U. Gilani ${ }^{1}$ \\ ${ }^{1}$ Mechanical Engineering Department, \\ Universiti Teknologi PETRONAS, Malaysia \\ ${ }^{2}$ Mechanical Engineering Department, College of Engineering, \\ Al-Nahrain University, Iraq
}

\begin{abstract}
The behaviour of artificially generated vortex by solar utilization is not well understood, as it is compressible, turbulent and 3-D. The present paper is aimed to simulate and analyse a new solar updraft power system, named vortex generation engine (VGE) to provide basic understanding of the artificial vortex. The main focus is on the effect of the upper plate diameter on the vortex structure. The simulation was carried out by CFD using ANSYS FLUENT 15. The modelled engine has $1.0 \mathrm{~m}$ outer diameter, $0.6 \mathrm{~m}$ height and 8 slots on the circumferential of the VGE for the air flow from the solar collector zone to the engine zone. The numerical procedure was validation by comparing the predicted temperature results with experimental measurements. The results have demonstrated that the upper hole of the VGE has considerable effect on the vortex generation. When the diameter of the upper hole increased from $0.3 \mathrm{~m}$ to $0.9 \mathrm{~m}$, the strength of the generated vortex decreased. The visualization through CFD simulation illustrates that a flow circulation exists at many regions in the engine body, which reduce the system performance. As the technique is new, there is a lot of room for further investigation and improvement of the design parameters of the proposed engine. Keywords: artificial vortex, solar updraft, solar vortex engine.
\end{abstract}

\section{Introduction}

The solar energy is the promising free-carbon source for world energy demand growth. Among the solar conversion systems, the natural solar updraft technique is demonstrated to be effective for power generation. The solar chimney, as solar 
updraft system, is the widely studied and investigated from many points of view of design, performance, and enhancement methods, as reported by Al-Kayiem and Aja [1]. The newly proposed solar updraft technique is the solar vortex technique, which is aimed to replace the tall chimney by artificially generated vortex. The principles and the attempts carried out; so far, to investigate the solar vortex engine (SVE) is summarized and reported by Mustafa et al. [2] and Mustafa et al. [3]. The SVE consists of three main parts; solar air collector (SAC), vortex generation engine (VGE) and wind turbine - generator unit for power generation, as shown in Figure 1.

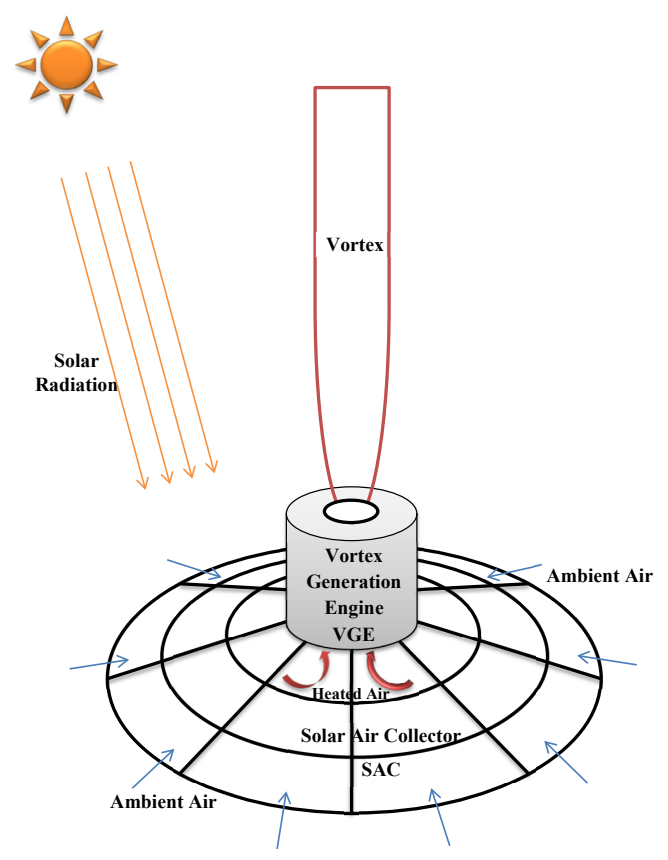

Figure 1: Schematic diagram of SVE, with main features of SAC and VGE.

Previous works have been carried out aiming to analyse and to understand the effect of design parameters on the behaviour the swirl updraft. Natarajan [4] performed numerical simulation of atmospheric vortex engine (AVE) by the CFD analysis. The results show that the AVE can generate a vortex flow in the atmosphere above the engine and the vortex acts as a physical chimney limiting the mixing of surrounding air into the raising plume of hot air. The temperature difference, $\Delta \mathrm{T}$ is the main parameter that controls the strength of the vortex and governs the power output. Then, Natarajan and Hangan [5] studied the effect of surface roughness on tornado-like vortices using Fluent 6.2 software. The investigations are carried out for swirl ratios between 0.1-2.0. They have claimed that introducing roughness increases the radial, axial and tangential velocities inside the core region. Wen and Bofeng [6] studied numerically the formation of helical vortices produced by the short twisted tape. The results showed that two 
symmetrical stable helical vortices are present downstream of the twisted tape. The intensities of helical vortices increase when increasing Reynolds numbers and the trend is in agreement with the swirl intensities. The intensities of helical vortices decay slowly compared with the intensities of swirling flow. Qian and Zhang [7] performed CFD numerical simulations of cyclones with various geometries and operating conditions to study the natural vortex length. The numerical solutions were carried out using commercial CFD code Fluent 6.1. The results show that inlet velocity, cyclone length and vortex finder insertion deepness also play important roles in influencing the natural vortex length other than the factors mentioned in publications. Kuai et al. [8], through CFD simulation, model to capture the flow characteristics of both full scale and laboratory-simulated tornadoes. It was found that the tornado is sensitive to the geometrical parameters used in the numerical model. Increased surface roughness was found to reduce the tangential speed in the vortex near the ground and enlarge the core radius of the vortex, which is in conflict with Natarajan [4] claims. Ishihara et al. [9] investigated flow fields of tornado-like vortices generated by a numerical tornado simulator using the LES turbulence model for two typical swirl ratios. The core radii of simulated vortices with swirl ratios of 0.31 and 0.65 showed favourable agreement with visualized vortices by a laboratory tornado simulator.

The literature is fairly demonstrating that many design parameters on the vortex generation system are not extensively investigated and analysed. Some works focused on the surface roughness and some works on the possible enhancement by insertion of twister. However, much more investigations are required to be carried out on the SVE to further understand the swirly flow due to temperature rise in the atmospheric air.

In this study, CFD technique by means of finite-volume technique has been used to model and simulate a VGE to investigate the thermo-hydrodynamic behaviour of vortex flow field, within the core of the VGE. The simulation was carried out under assumptions of compressible, steady, turbulent, and 3-D flow. The main focus was on the effect of the size of the upper hole of the cover plate of the VGE. Upper hole diameters of 0.3 and $0.9 \mathrm{~m}$ were considered and the flow field was characterized in terms of the vortex strength. Moreover, model validation with experimental measurements.

\section{Numerical simulation}

\subsection{Model configuration and mesh generation}

The modelling is carried out in GAMBIT and subsequently the mesh is imported to FLUENT for solving and post processing as $3 \mathrm{D}$ cylindrical problem. The VGE configuration has an inner cylinder with eight rectangular entry air slots, eight curved air guide vanes, $\mathrm{N}$, circular top hole, and outer cylinder embracing all the said parts (Figure 2). The tangential flow has been produced by the guide vanes within convergence region. The adopted dimensions of the VGE model are; the radius of inner cylinder is $0.45 \mathrm{~m}$ with height of $0.6 \mathrm{~m}$, and outer cylinder of radius 
of $0.5 \mathrm{~m}$ with height of $0.6 \mathrm{~m}$. The deflection angle of the curved air guide vanes is set to $\delta=25^{\circ}$. An upper hole of $0.3 \mathrm{~m}$ diameter was used for the swirly flow exit, similar to the experimental model.

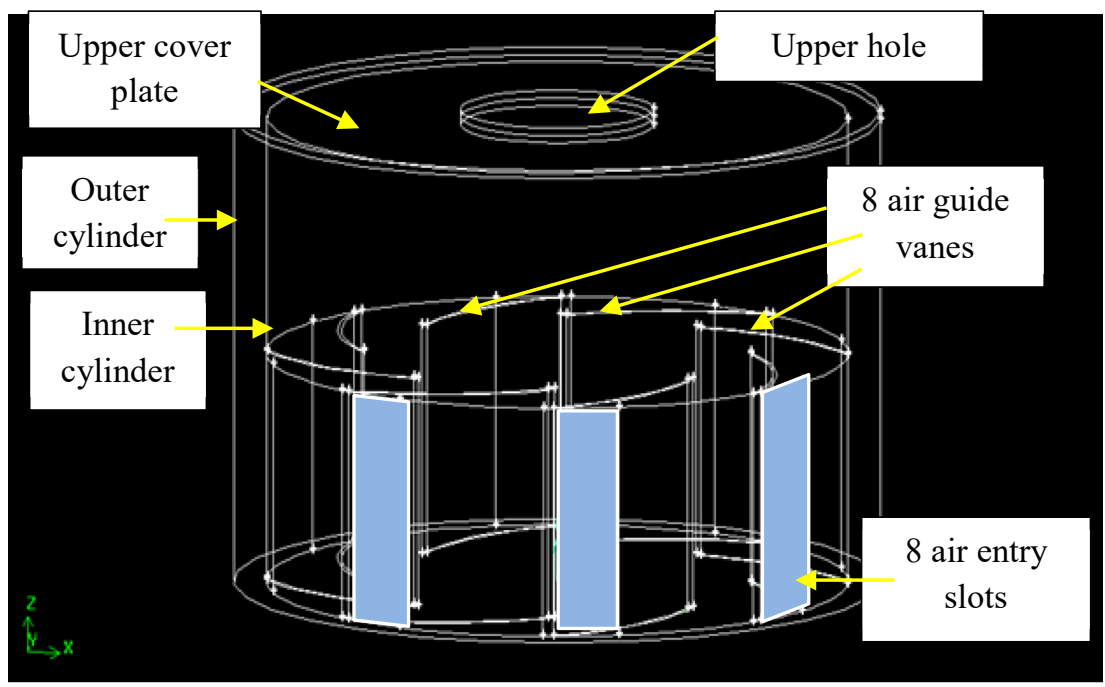

Figure 2: Configuration of the VGE model.

Numerical simulation of swirling flow needs high quality with mesh gradient toward the centre, selection of grid interval for mesh depends on size and complexity degree. For the VGE model and the domain, by comparing the mesh parameters of EquiSize Skew and Aspect ratio, size interval of $0.01 \mathrm{~m}$ has been selected for good mesh properties with 272364 computational cells. Mesh of the VGE model was carried out by using tetrahedral elements of T-grid scheme. Then, the mesh was written out in the format used by FLUENT for solving process.

\subsection{CFD procedure}

The values of the input parameters, which are the temperature and the flow velocity, to the VGE are obtained from the SAC measured data. Experimental parameters at entry slot of the VGE have been measured, with velocity of $0.5 \mathrm{~m} / \mathrm{s}$ and temperature of $31^{\circ} \mathrm{C}$, and used as boundary conditions for the simulation. The ambient conditions surrounding the SVE imply that air density and viscosity are $1.161 \mathrm{~kg} / \mathrm{m}^{3}$ and $1.846 \times 10^{-5} \mathrm{~kg} / \mathrm{m}$-s, respectively, at average temperature of $26^{\circ} \mathrm{C}$. The numerical simulation of the VGE was validated through comparison with the experimental measurements. Once validation was achieved, the simulation has been carried out for various diameters of the top plate. The basic diameter is $300 \mathrm{~mm}$ and then increased to $900 \mathrm{~mm}$ to inspect the influence on the vortex structure. 


\subsection{Numerical simulation methods}

The VEG model and its domain were simulated in steady, compressible, turbulent and viscous flow field. Numerical method employed for CFD simulation of the VGE model consist of "pressure - based" solver applied in solution, "pressure velocity coupling" scheme used in solving the flow problem in a segregated manner, and advanced viscous RNG k- $\varepsilon$ model. RNG k- $\varepsilon$ viscous model and the turbulent viscosity modification for the swirl effects are enabled to simulate the vortex field generated by VGE.

\section{Results and discussion}

Results of temperature, pressure, and velocity within the VGE flow field will be presented and discussed. Numerical results have been compared with measured values at ambient temperature, $T_{a m b}$ of $35^{\circ} \mathrm{C}$, input air temperature to the VGE of $41^{\circ} \mathrm{C}$, and input air velocity at inlet slots of $0.5 \mathrm{~m} / \mathrm{s}$.

\subsection{Analysis of temperature distribution}

Results of total temperature distribution are shown in Figure 3 for case (a), where the upper hole diameter is $0.3 \mathrm{~m}$ and (b), where the upper hole diameter is $0.9 \mathrm{~m}$. The simulation results show that, the temperature of air within the engine body is decreasing from the side walls towards the centre.

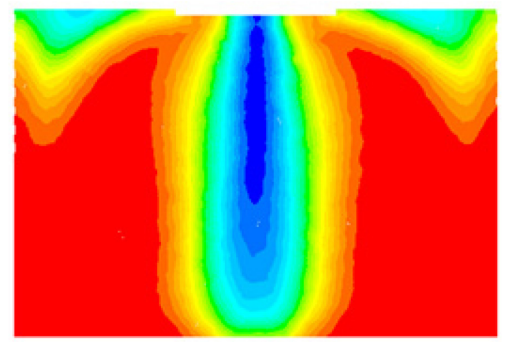

(a) $\mathrm{D}_{\mathrm{h}}=0.3 \mathrm{~m}$

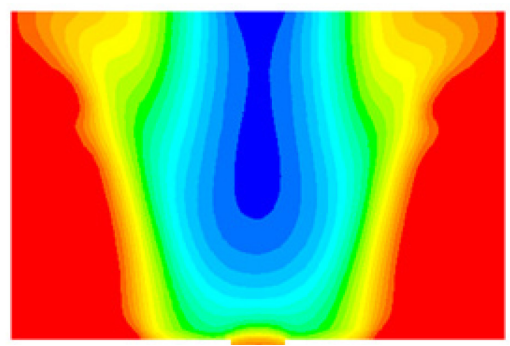

(b) $\mathrm{D}_{\mathrm{h}}=0.9 \mathrm{~m}$

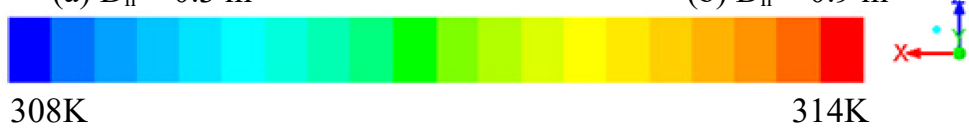

Figure 3: Contours of temperature distribution in $(\mathrm{K})$ within the VGE for air properties at entry slot of $\mathrm{V}=0.5 \mathrm{~m} / \mathrm{s}$ and $\mathrm{T}=314 \mathrm{~K}$; the upper hole diameter of (a) $0.3 \mathrm{~m}$ and (b) $0.9 \mathrm{~m}$.

Further, the temperature decreases close to the upper cover, and temperature gradation focused toward the upper hole in the centre of the upper cover. Simulation case (a) indicates that the lowest temperature at the centre line of the VGE which it's equal to input temperature, the lowest temperature indicates to the 
eye of natural vortex. Case (b) has the same gradation trend as in (a), but in large temperature gradient because the upper hole diameter of $0.9 \mathrm{~m}$ permits the flow to exit with lower residence time inside the engine.

\subsection{Analysis of axial velocities}

The velocity results are presented as vectors for the axial components and tangential component within the VGE. Vectors results in vertical cross section, shown in Figure 4, display the flow axial velocity structure. Simulation case (a) shows the direction of air entering the slots as dots vectors, and air movement as slope vectors in the convergence region.

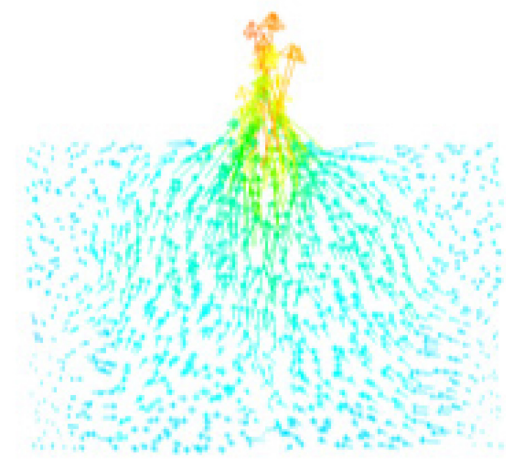

(a) $\mathrm{D}_{\mathrm{h}}=0.3 \mathrm{~m}$

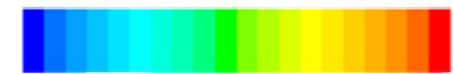

$-1.1 \mathrm{~m} / \mathrm{s}$
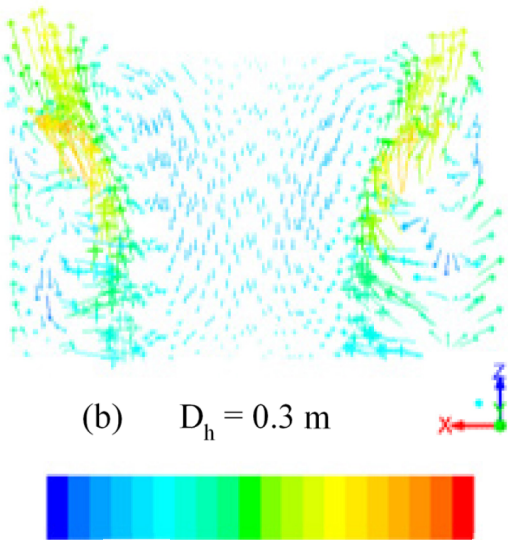

$-0.4 \mathrm{~m} / \mathrm{s}$

Figure 4: Vectors of axial velocity distribution in $(\mathrm{m} / \mathrm{s})$ within the VGE for air properties at entry slot of $\mathrm{V}=0.5 \mathrm{~m} / \mathrm{s}$ and $\mathrm{T}=314 \mathrm{~K}$; the upper hole diameter of (a) $0.3 \mathrm{~m}$ and (b) $0.9 \mathrm{~m}$.

Most of air motion shown in case (a) has been moved from entry slots toward the upper hole, with weak circulation region in the corners. While case (b) shows vectors motion in an irregular direction at entry slots with large circulation bubbles. This is an indication for the poor design of the slot. Larger area of the slots will reduce the circulation and reduce the blockage of the entire flow from the exit of the SAC to the body of the engine. Insight analysis of the flow behaviour summarized by the two figures indicates that the upper plate plays a role in the produced structure and strength of the vortex. In case (a), the flow is mainly directed to the central part with high vertical component, but small vortex size at the zone just upper the plate. In case (b), the flow is sound to direct to the circumferential of the engine and leaves with larger zone of rotation. The reason of the flow circulation motion dates back to increasing diameter of the upper hole from $0.3 \mathrm{~m}$ to $0.9 \mathrm{~m}$. For no doubt, the presence of any sort of circulation within 
the system represents a weakness. However, fortunately, the present simulation permits good estimation on the engine design in terms of the slots and the size of the hole in the upper plate.

\subsection{Analysis of tangential velocities}

Figure 5 shows the predicted tangential velocity distribution at the top section of the engine core, i.e. velocity vectors field in a horizontal plane at $0.6 \mathrm{~m}$ height within VGE. The figure shows the tangential velocity components for cases (a) and (b). In both cases, upper hole diameter of $0.3 \mathrm{~m}$ and $0.9 \mathrm{~m}$, the tangential velocity has same sequence in growth within the convergence region. The tangential velocity was grown in vertical direction toward upper hole of $0.3 \mathrm{~m}$ diameter, while the tangential velocity has been decreased toward upper hole of $0.9 \mathrm{~m}$ diameter. The significant difference between the two cases that the tangential velocity component, in $0.3 \mathrm{~m}$ case, increase considerably within the central part of the engine. In contrast, the tangential velocity components, in the case of $0.9 \mathrm{~m}$ upper hole, are increasing at the outer zone of the engine. In fact, which is more effective in the system performance is not well understood. Further analyses are needed to study the back effect on the up draft of air, and more analysis on the produced pressure difference.

\subsection{Validation of the CFD simulation}

Validation of the numerical procedure has been achieved by comparison the simulation results with the experimental results obtained from the measurements of experimental model. The numerically predicted and the measured air temperatures, of the $0.3 \mathrm{~m}$ hole diameter case, have been compared, as in Figure 6 . The mean percentage of differences between the simulation results and the experimental measurement is $5.1 \%$, which is acceptable for such complex flow phenomena.

The results in the figure demonstrate that the CFD simulation results of the temperature are lower than the measured ones. The reason dates back to the existence of additional heat sources in the experimental work which have not been accounted in the CFD simulation. This additional heat is created from the upper plate and transfer to air in the engine core in terms of convection and radiation heat.

\section{Conclusions}

VGE is the heart part of the SVE. The flow field within the VGE is simulated computationally, using ANSYS FLUENT 15, and the results are validated by comparison with experimental measurements. The conclusions drawn the results, are:

- The obtained results are clearly showed the capability of the proposed VGE to produce artificial vortex by the solar energy utilization. 

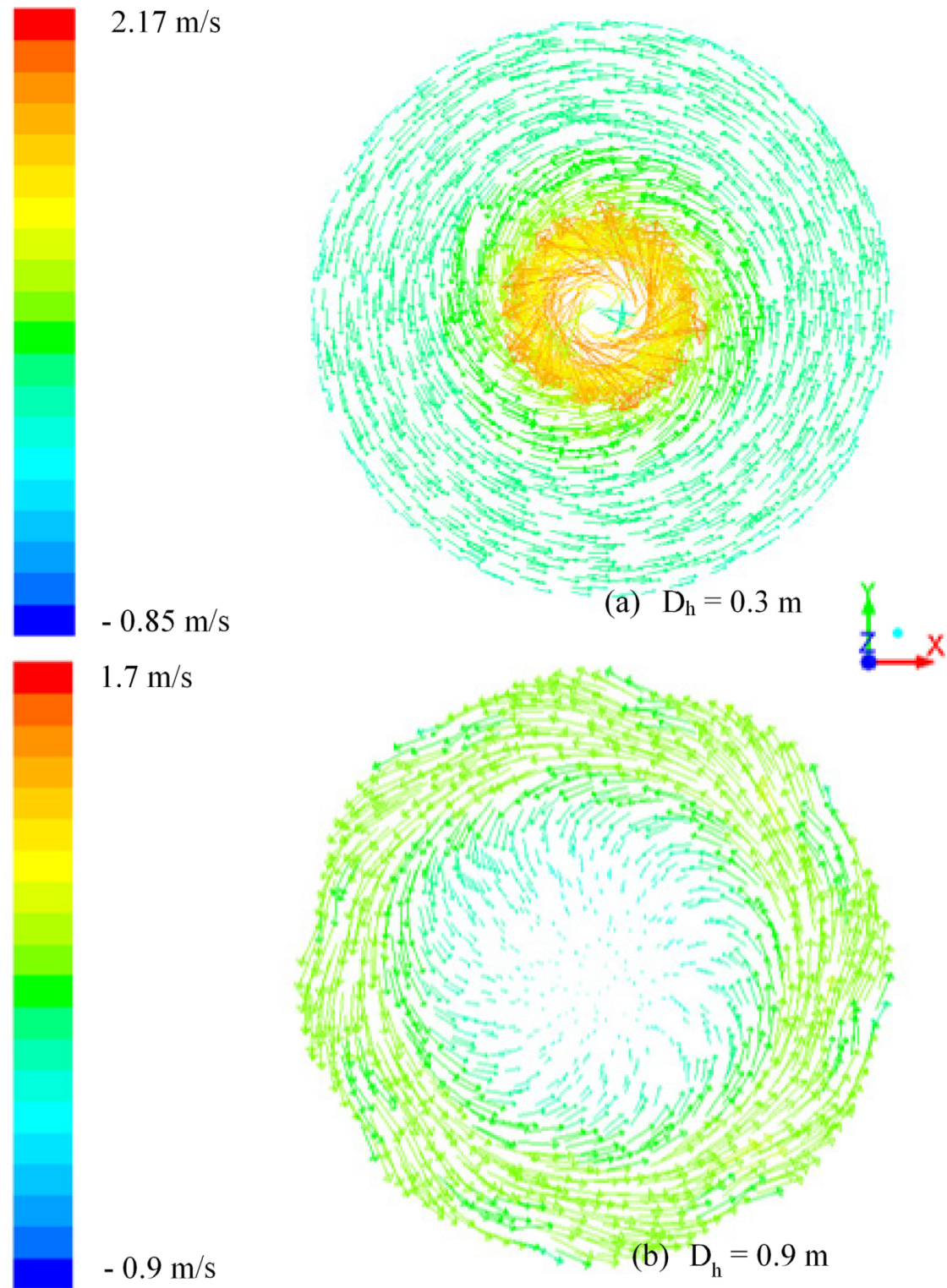

Figure 5: Vectors of tangential velocity distribution in $(\mathrm{m} / \mathrm{s})$ within the VGE for air properties at entry slot of $\mathrm{V}=0.5 \mathrm{~m} / \mathrm{s}$ and $\mathrm{T}=314 \mathrm{~K}$; the upper hole diameter of (a) $0.3 \mathrm{~m}$ and (b) $0.9 \mathrm{~m}$; at (1) engine base, (2) $0.3 \mathrm{~m}$ height, and (3) $0.6 \mathrm{~m}$ height. 


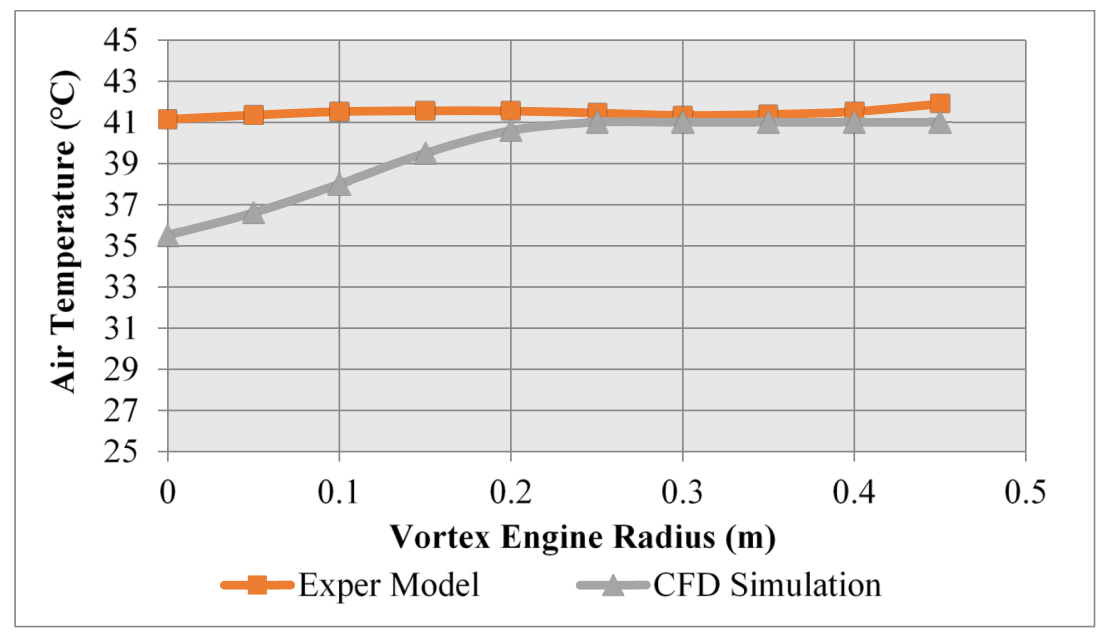

Figure 6: Validation of air temperature within the VGE.

- The tangential velocity is growing in value in the upper zone of the engine, compared to the lower zone, in the case of $0.3 \mathrm{~m}$ diameter, while the tangential velocity is decreasing is the flow moves up, in the case of $0.9 \mathrm{~m}$ upper hole diameter.

- The upper hole of the VGE has considerable affects the vortex generation and swirl strength. When the diameter of the upper hole increases from $0.3 \mathrm{~m}$ to $0.9 \mathrm{~m}$, the strength of the generated vortex decreases, which is indicated by the reduced values of the tangential velocity component.

However, it is recommended to extend the simulation and the experimental measurement to inspect the back effect of the upper hole on the up drafting force. This requires further simulation and analysis in terms of the pressure and pressure difference across the engine.

\section{Acknowledgements}

The authors would like to acknowledge Universiti Teknologi PETRONAS for the financial, technical and logistic support to produce the work under the University fund, i-Gen 0153DA-069. The second author, in particular, would like to express thankful remarks to UTP for supporting his PhD study under the Tuition Fees Assistance (TFA) scheme.

\section{References}

[1] Al-Kayiem, H.H. and Aja, O.C., Historic and Recent Progress in Solar Chimney Power Plant Enhancing Technologies, Renewable and Sustainable Energy Reviews, Vol. 58, pp. 1269-1292. 2016. DOI: 10.1016/ j.rser.2015.12.331. 
[2] Mustafa, A.T., Al-Kayiem, H.H. and Gilani, S.I.G., A Review of Convective and Artificial Vortices for Power Generation, Int. J. Sus. Dev. Plann., Vol. 10, No. 5, pp. 650-665. 2015. DOI: 10.2495/SDP-V10-N5-650665.

[3] Mustafa, A.T., Al-Kayiem, H.H. and Gilani, S.I.G., A Survey on Performance Enhancement of Solar Updraft Tower Power Plants, Int. J. of Eng. And Technical Research, Vol. 2, No. 7, pp. 34-39. 2014.

[4] Natarajan, D., Numerical Simulation of Tornado-like Vortices, PhD, Civil and Environmental Engineering, University of Western Ontario, London, Ontario, Canada. 2011.

[5] Natarajan, D. and Hangan, H., Numerical Study on the Effects of Surface Roughness on Tornado-like Flows, in 11th Americas Conference on Wind Engineering (11ACWE). 2009.

[6] Wen, L. and Bofeng, B., A numerical study on helical vortices induced by a short twisted tape in a circular pipe, Case Studies in Thermal Engineering, Vol. 5, pp 134-142. 2015.

[7] Qian, F. and Zhang, M., Study of the Natural Vortex Length of a Cyclone with Response Surface Methodology, Computers \& Chemical Engineering, Vol. 29, pp. 2155-2162. 2005.

[8] Kuai, L., Haan Jr, F.L., Gallus Jr, W.A. and Sarkar, P.P., CFD Simulations of the Flow Field of a Laboratory-simulated Tornado for Parameter Sensitivity Studies and Comparison with Field Measurements, Wind and Structures, Vol. 11, pp. 75-96. 2008.

[9] Ishihara, T., Oh, S. and Tokuyama, Y., Numerical Study on Flow Fields of Tornado-like Vortices using the LES Turbulence Model, Journal of Wind Engineering and Industrial Aerodynamics, Vol. 99, pp. 239-248. 2011. 